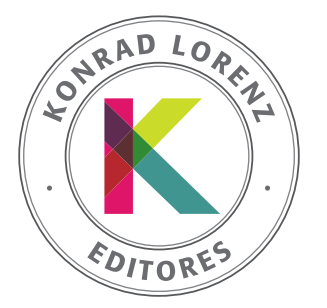

\title{
Construcción y validación del Repositorio de Expresiones Faciales Emocionales Colombianas
}

\author{
Sofía Zapata-Orozco ${ }^{\mathrm{a}}$, José Zapata-González ${ }^{\mathrm{a}}$, Carlos Gantiva ${ }^{\mathrm{b}}$, \\ Paola Suárez-Pico ${ }^{c}$ y Mauricio Barrera-Valencia ad*
}

\author{
a Universidad de Antioquia, Medellín, Colombia \\ ${ }^{b}$ Universidad de los Andes, Bogotá, Colombia \\ ' Fundación Universitaria Konrad Lorenz, Bogotá, Colombia

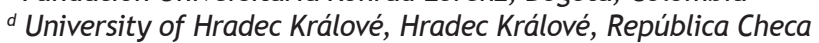

Recibido el 9 de diciembre de 2019; aceptado el 14 de septiembre de 2020

\author{
PALABRAS CLAVE \\ Rostros, \\ emociones, \\ reconocimiento \\ emocional, \\ validación, \\ Colombia
}

\begin{abstract}
Resumen Los rasgos fenotípicos afectan la respuesta hacia los rostros emocionales. En Colombia, no se ha construido y validado un banco de imágenes de rostros emocionales. El objetivo del presente estudio fue construir y validar un banco de fotografías de rostros emocionales colombianos (alegría, ira, tristeza, miedo, asco, sorpresa y expresión neutral/descanso), a través de una metodología similar a la utilizada en el diseño de otros bancos de expresiones emocionales. El estudio se llevó a cabo con 80 participantes (43 mujeres), a los cuales se les presentó cada una de las 70 expresiones emocionales del Repositorio de Expresiones Faciales Emocionales Colombianas (REFEC). A partir de estudios previos en este campo se tomaron como índices de validez el porcentaje de acierto y el coeficiente de kappa de Fleiss (grado de concordancia entre evaluadores). Además, se midió el tiempo de reacción como índice de demanda cognitiva para el reconocimiento de la expresión emocional. Los resultados muestran un porcentaje de acierto superior al de otros bancos de estímulos de expresiones emocionales y un índice de concordancia bueno/excelente (similar al de otros instrumentos). En conjunto, los resultados sugieren que el REFEC es un banco de imágenes de expresiones faciales emocionales válido para su uso en población colombiana.
\end{abstract}

(c) 2020 Fundación Universitaria Konrad Lorenz. Este es un artículo Open Access bajo la licencia CC BY-NC-ND (http://creativecommons.org/licenses/by-nc-nd/4.0/).

\section{Design and validation of the Colombian Repository of Emotional Facial Expressions}

\section{KEYWORDS}

Faces, emotions, emotional recognition, validation, Colombia
Abstract Phenotypic traits affect the response to emotional faces. In Colombia, an image bank of emotional faces has not been built and validated. The objective of this study was to build and validate a bank of photographs of Colombian emotional faces (joy, anger, sadness, fear, disgust, surprise and neutral expression/rest), through a methodology similar to that used in the design of other banks of emotional expressions. The study was carried out with 80 participants (43 women), to whom each of the 70 emotional expressions of the Repository of

\footnotetext{
* Autor para correspondencia.
}

Correo electrónico: mauricio.barrera@udea.edu.co 
Colombian Emotional Facial Expressions (REFEC) was presented. From previous studies in this field, the percentage of correctness and the Fleiss kappa coefficient (degree of agreement between evaluators) were taken as validity indices. Additionally, reaction time was measured as an index of cognitive demand for the recognition of emotional expression. The results show a percentage of correctness higher than that of other stimulus banks of emotional expressions and a good / excellent concordance index (similar to that of other instruments). Together, the results suggest that REFEC is a valid image bank of emotional facial expressions for use in the Colombian population.

(c) 2020 Fundación Universitaria Konrad Lorenz. This is an open access article under the CC BYNC-ND license (http://creativecommons.org/licenses/by-nc-nd/4.0/).

Las expresiones faciales dan cuenta del estado emocional de la persona. Su adecuado reconocimiento permite reaccionar de forma adaptativa, lo que lo convierte en un indicador del desempeño esperado de un individuo en el ámbito social (Jeanneret, Oña, Rego, Vaiman \& Pereno, 2015). El estudio del reconocimiento de las expresiones faciales ha sido un campo de amplio desarrollo en la psicología y las neurociencias. Las investigaciones relacionadas con el reconocimiento y procesamiento de rostros se han desarrollado en áreas como la esquizofrenia (Quintero et al., 2015), el autismo (Suárez-Pico, Bonelo-Cuellar \& Utria, 2019). la demencia frontotemporal (Narambuena, Vaiman \& Pereno, 2016) y el procesamiento cortical (Gantiva, Sotaquirá, Araujo \& Cuervo, 2020), entre muchas otras.

A partir de los postulados darwinianos sobre la universalidad de las emociones y su desarrollo evolutivo, y los estudios pioneros de Ekman y Friesen (1971), se propone que las emociones y su expresión facial tienen un origen transcultural (Gantiva et al., 2019). Se identifican seis emociones básicas (i.e., alegría, ira, tristeza, sorpresa, asco y miedo), las cuales están presentes de manera similar en todas las culturas observadas (Ekman, 1993a).

A pesar del origen evolutivo y transcultural de las emociones, recientemente ha surgido evidencia que sugiere la existencia de factores particulares en cada grupo humano, como el sexo, la emoción específica, la edad, los rasgos fenotípicos faciales asociados a diferentes etnias y el énfasis dado culturalmente a un rasgo u otro, que afectan la precisión en el reconocimiento de la emoción expresada en el rostro (Chen \& Jack, 2017; Barrett, Adolphs, Marsella, Martinez \& Pollak, 2019). Por esta razón, se han desarrollado instrumentos compuestos por bancos de imágenes de expresiones faciales validados en diferentes países; por ejemplo, el Facial Expressions of Emotion - Stimuli and Tests (FEEST, 60 rostros) (Young, Perrett, Calder, Sprengelmeyer \& Ekman, 2002). El NimStim Emotional Face Smuli (672 rostros) (Tottenham et al., 2009), el Karolinska Directed Emotional Face (KDEF, 70 rostros) (Lundqvist, Flykt \& Öhman,1998), el Pictures of Facial Affect (POFA, 110 rostros) (Ekman, 1993b), y el Japanese and Caucasian Facial Expressions of Emotion (JACFEE) (56 rostros) (Matsumoto \& Ekman, 1988).

En Colombia no existen instrumentos válidos y confiables que permitan evaluar el reconocimiento de expresiones faciales emocionales. Torrado, Ornar, Prada, Edward y Santos (2012), realizaron una prueba piloto como intento de validación de instrumentos extranjeros. Sin embargo, los resultados sugieren que las fotografías utilizadas contienen rasgos fenotípicos y problemas asociados con la calidad de las fotos que dificultan su uso en población colombiana. Por lo anterior, el objetivo del presente estudio fue construir y validar, a través de una metodología similar a la utilizada en el instrumento NimStim Emotional Face Smuli (Tottenham et al., 2009), un repositorio de imágenes de expresiones faciales con contenido emocional, desarrollado con rasgos fenotípicos (rostros) de personas colombianas.

\section{Método}

\section{Participantes}

La muestra estuvo conformada por 80 colombianos mayores de edad (43 mujeres y 37 hombres), con una edad promedio de 22 años $(D E=3.3)$. Los participantes recibieron información oral y escrita sobre los objetivos del estudio, las implicaciones de hacer parte de la investigación, la protección de sus datos personales y la posibilidad de retirarse en cualquier momento. El estudio cumplió con los estándares éticos de la Convención de Helsinki (World Medical Association Declaration of Helsinki, 2000) y fue aprobado por el Comité del Centro de Investigaciones de la Facultad de Ciencias Sociales y Humanas de la Universidad de Antioquia (Colombia).

\section{Estímulos}

Para el diseño de las expresiones faciales se realizó, en una primera etapa, una convocatoria abierta a través de redes sociales para personas que tuvieran habilidades para expresar facialmente emociones de forma voluntaria. Además, debían haber nacido en Colombia, ser hijos de padres colombianos, no haber vivido en el exterior por más de cinco años y contar con alguna experiencia en actuación o modelaje. Se presentaron 80 personas de las cuales se descartaron 64 por no vivir en la ciudad en donde se realizaría la sesión de fotos, esperar retribución económica, presentar modificaciones estéticas en el rostro o no contar con experiencia en actuación o modelaje. Se realizaron 16 pruebas por video y de ahí se descartaron cuatro personas por mostrar un pobre desempeño en las tareas solicitadas o no seguir las instrucciones dadas. Al final, se conformó un grupo de 13 modelos ( 7 mujeres y 6 hombres) con una mediana de edad de 22 años $(R I Q=12)$.

Después de brindar su consentimiento informado para el uso de su imagen con propósitos investigativos, los 13 modelos asistieron a una sesión fotográfica de 45 minutos 
en un ambiente controlado, en el que debían recoger su cabello, limpiarse la cara y retirarse el maquillaje y los accesorios. Con el fin de unificar las condiciones para la toma de las fotos, todos los participantes emplearon una camiseta de color negro y cuello redondo. Las imágenes se tomaron con fondo blanco y con una distancia de dos metros con respecto a la cámara. La altura de la cámara se ajustó a la altura del rostro de cada sujeto. Todas las tomas emplearon un lente $18-55 \mathrm{~mm}$, un flash básico con ayuda de un difusor y una pantalla de rebote para suavizar las sombras. Las fotos obtenidas fueron de alta resolución y en formato .raw, en la figura 1 se presentan algunos ejemplos de las fotografías tomadas.

A los modelos se les solicitó recordar situaciones relacionadas con cada una de las emociones y representar las emociones de alegría, ira, tristeza, miedo, asco y sorpresa, más una expresión neutra o de descanso, siguiendo el modelo de Ekman y Cordaro (2011). En promedio, se obtuvieron seis fotografías por emoción por cada modelo. Posterior a una revisión técnica de las fotografías obtenidas, se eliminaron aquellas que estaban desenfocadas, movidas, mal iluminadas o con elementos intrusos en el cuadro, para conformar así un banco de 216 fotografías.

En una segunda etapa, se seleccionaron cinco jueces expertos colombianos (formación en psicología o ciencias afines, con experiencia clínica o investigativa de al menos tres años en campos relacionados con el estudio de las emociones y con título de maestría o doctorado), para realizar una validación de contenido de las 216 fotografías. Cada foto se presentó individualmente a cada juez y se le solicitó calificarla en tres dimensiones: emoción, facilidad de reconocimiento e intensidad de la emoción. Para la dimensión de emoción, debía marcar por cada imagen una sola opción entre alegría, asco, ira, miedo, sorpresa, tristeza, descanso y ninguna de las anteriores. La dimensión facilidad de reconocimiento se evaluó en una escala de 1 a 5 , en donde 1 era "muy difícil" y 5 "muy fácil". La dimensión intensidad de la emoción se evaluó también en una escala de 1 (muy sutil) a 5 (muy intensa).

Debido a que participaron más de dos jueces, se utilizó el coeficiente kappa de Fleiss para establecer el grado de concordancia entre evaluadores (Fleiss, 1981; (Sharma, Desikachari \& Sarma, 2019), siguiendo los parámetros de estudios recientes en donde hay más de dos opciones de respuesta (Wilks et al., 2019). Para la selección del grupo final de fotografías se tomaron en cuenta puntajes en el coeficiente kappa de Fleiss superiores a 0.74 (entre bueno y excelente, según la denominación dada por Almanasreh, Moles y Chen, 2019 y Polit et al., 2007), y puntajes iguales o superiores a 3 en facilidad. La variable intensidad no se tuvo en cuenta debido a que las expresiones de descanso siempre tuvieron bajo puntaje en esta variable. A partir de estos criterios, se seleccionaron las diez expresiones con mejores puntajes por cada emoción (10 fotografías, cinco de mujeres y cinco de hombres, para cada una de las siete expresiones emocionales), para un total de 70 fotografías.

En general, las 70 imágenes seleccionadas tuvieron una concordancia entre jueces casi perfecta (coeficiente kappa de Fleiss $=.96, D E=.08$ ) y un nivel alto de facilidad $(M=4.11, D E=.49)$. Sin tener en cuenta las expresiones de descanso, la intensidad fue, en general, media alta $(M=3.71, D E=.68)$. No se encontraron diferencias significativas entre el sexo del modelo en ninguna variable (todas las $p>$.21). Las 70 expresiones emocionales del Repositorio de Expresiones Faciales Emocionales Colombianas (REFEC), se pueden descargar libremente en: http://bibliotecadigital.udea.edu.co/handle/10495/16366

\section{Procedimiento}

Las imágenes se presentaron a través de una tarea de reconocimiento facial, diseñada en el software PsychoPy v1.8 (Peirce, 2009). Esto permitió estandarizar las condiciones de presentación, aleatorizar el orden de las imágenes y medir los tiempos de reacción. La tarea consistió en presentar cada fotografía en una pantalla plana de 19 pulgadas, luego, el participante debía identificar y seleccionar la emoción que estaba expresando ese rostro. La selección se reportaba presionando una tecla en el computador. A partir de los criterios de Young et al. (2002), la duración de cada imagen fue de 5 segundos, se presentaron de forma aleatoria y el participante no tenía límite de tiempo para responder.

Cuando el rostro desaparecía, se reemplazaba la imagen por las claves de las teclas que se debían oprimir para cada emoción. Una vez el participante presionaba una tecla, aparecía la siguiente fotografía y continuaba con este proceso hasta completar los 70 estímulos (ver figura 2). Para
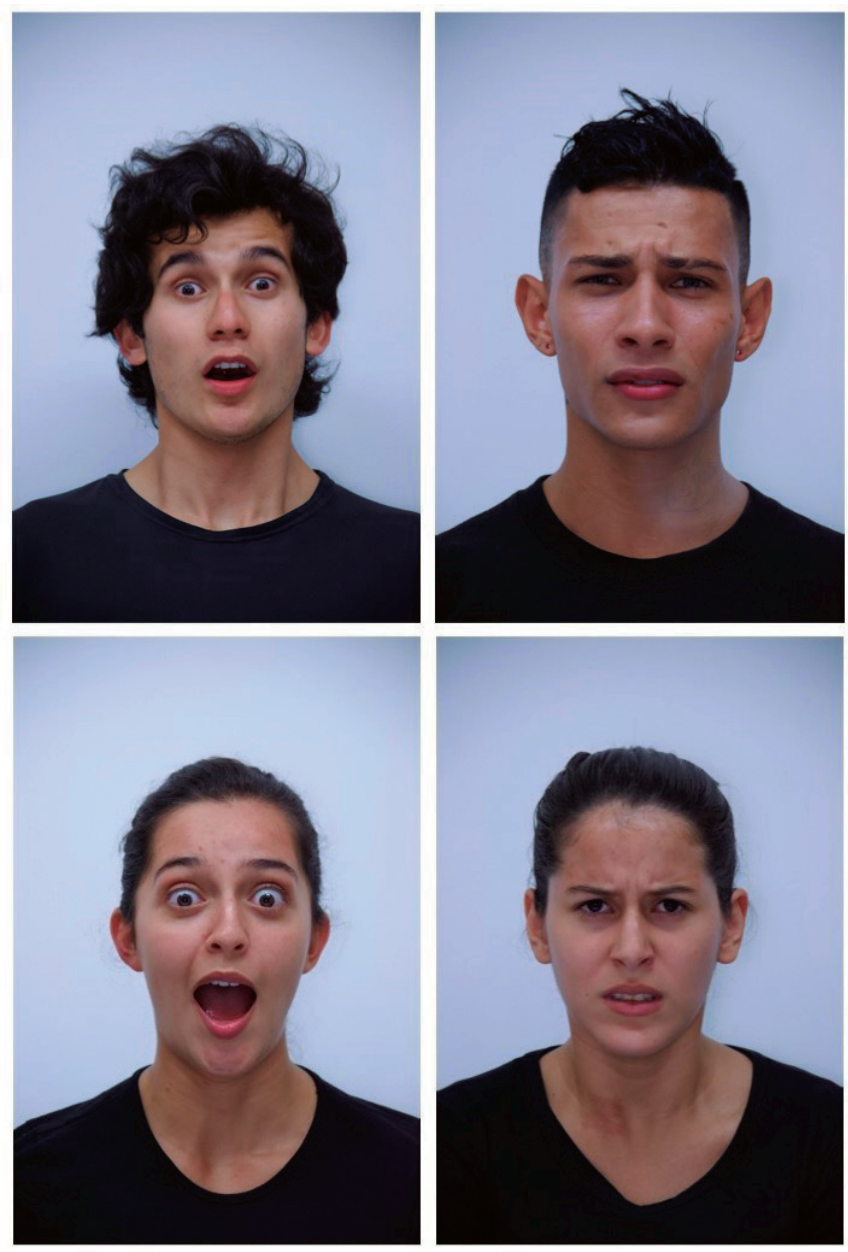

Figura 1. Ejemplos de algunas de las expresiones faciales realizadas por los modelos 

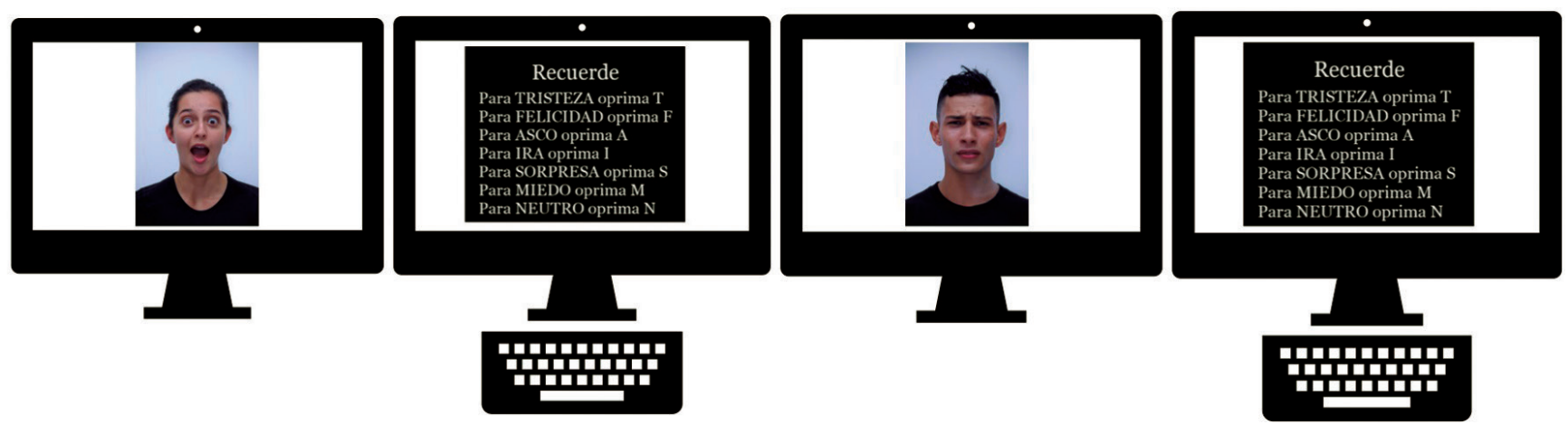

Figura 2. Esquema del procedimiento empleado

asegurarse de que cada participante había comprendido el procedimiento de la tarea, se hizo un entrenamiento previo con siete fotografías del repositorio KDEF (Lundqvist et al., 1998), correspondientes a cada una de las siete expresiones emocionales.

\section{Análisis estadístico}

Para establecer las diferencias en el porcentaje de acierto de las respuestas y tiempos de reacción, se aplicó un ANOVA mixta de medidas repetidas $2 \times 2 \times 7$. Con el factor intersujeto Grupo (sexo del participante, hombre o mujer) y con los factores intrasujeto Modelo (sexo del modelo, hombre o mujer) y Emoción (alegría, miedo, ira, tristeza, asco, sorpresa y descanso). Para establecer las diferencias en el coeficiente kappa de Fleiss (Fleiss, 1981) entre las diferentes expresiones, se aplicó un ANOVA 2 (Modelo) $\times 7$ (Emoción). Las comparaciones múltiples de pares post hoc se hicieron con la corrección Bonferroni (Bland \& Altman, 1995). El tamaño del efecto se reporta para cada ANOVA. Finalmente, se realizó un análisis de correlación de Pearson entre porcentaje de acierto de la respuesta y el tiempo de reacción. El nivel de significancia para todos los análisis se estableció en $p<.05$ y se realizaron con el programa SPSS 20.0.

\section{Resultados}

\section{Validez}

Se analizaron dos medidas de validez (porcentaje de acierto y el coeficiente kappa de Fleiss), para cada imagen. En el material suplementario (S1), disponible en línea, se describen estas medidas para cada modelo y expresión emocional. En general, el porcentaje de acierto fue alto (Media $=80.92, D E=8.46$, Mediana $=81.42)$ y el coeficiente kappa de Fleiss fue bueno/excelente (Polit et al., 2007) $($ Media $=.72, D E=.21$, Mediana $=.79)$. En la figura 3 , se presentan estas medidas de validez y el tiempo de reacción para cada emoción.

El ANOVA para la variable porcentaje de acierto identificó un efecto principal significativo para Emoción $\left(F_{6,468}\right.$ $\left.=88.71, p<.001, \eta_{p}{ }^{2}=.53\right)$. Las expresiones de descanso, alegría y sorpresa tuvieron mayores porcentajes de acierto en comparación con el resto de emociones (todas las $p<.001$ ). Las expresiones de ira y asco tuvieron mayores porcentajes de acierto que las de tristeza y miedo (todas las $p<.006)$, y la expresión de tristeza mayor porcentaje de acierto que la de miedo $(p<.001)$. El factor Grupo fue significativo $\left(F_{1,78}=10.53, p=.002, \eta_{p}^{2}=.11\right)$, en general, las mujeres tuvieron mayor porcentaje de acierto

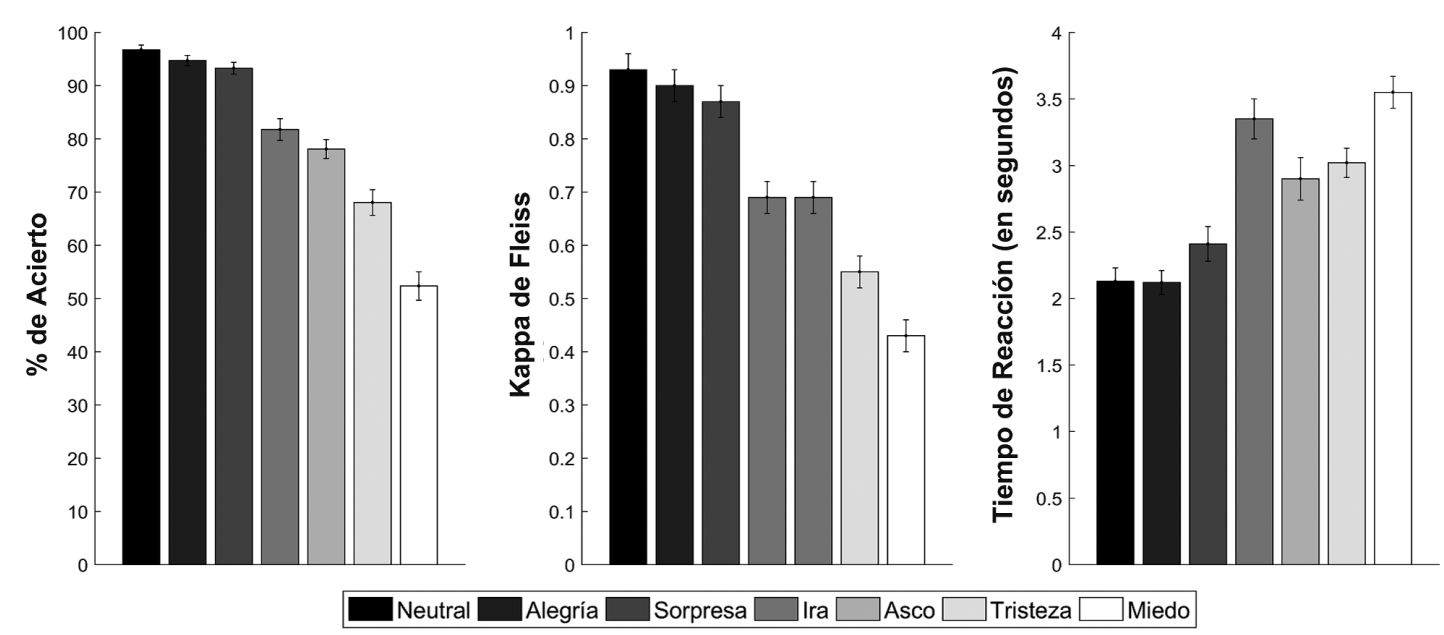

Figura 3. Porcentaje de acierto, coeficiente kappa de Fleiss y promedio de tiempo de reacción para cada expresión emocional. Las barras indican \pm 1 error estándar de la media 
$(M=83.62, D E=6.40)$ (i.e., mejor reconocimiento emocional), que los hombres $(M=77.79, D E=9.52)$. El factor Modelo también fue significativo $\left(F_{1,78}=17.52, p<.001, \eta_{p}{ }^{2}=.18\right)$, las mujeres modelos generaron mayor porcentaje de acierto $(M=82.67, D E=9.49)$ que los modelos hombres $(M=78.74$, $D E=9.41)$. Sin embargo, como se identifica en el efecto de interacción significativo Emoción $\times$ Modelo $\left(F_{6,468}=11.31\right.$, $p<.001, \eta_{p}{ }^{2}=.12$ ), fue en las expresiones de sorpresa, asco y miedo en donde las modelos mujeres produjeron mayores porcentajes de acierto (todas las $p<.03$ ), mientras que en la expresión de ira los modelos hombres generaron mayor porcentaje de acierto $(p<.001)$. En la expresión de alegría se encontró una diferencia marginalmente significativa a favor de las modelos mujeres ( $p=.07$ ).

El ANOVA para el coeficiente kappa de Fleiss encontró un efecto principal significativo para Emoción $\left(F_{6,56}=24.12\right.$, $p<.001, \eta_{p}{ }^{2}=.72$ ). Las expresiones de descanso, de alegría y sorpresa tuvieron mayores puntajes en el coeficiente en comparación con el resto de emociones (todas las $p<.03$ ), y las expresiones de ira y asco mayores puntajes que las de miedo (ambas $p<.001$ ).

En la tabla 1, se presenta la matriz de confusión para las elecciones de los participantes, la cual representa el porcentaje promedio de aciertos y no aciertos para cada una de las expresiones emocionales (Tottenham et al., 2009), de esta forma, se puede observar el porcentaje con el que cada emoción fue confundida con otra y también el porcentaje de acierto. Las emociones que con mayor frecuencia se confunden son miedo con sorpresa (27\%), asco con ira (14.62\%), tristeza con miedo (12.37\%), miedo con tristeza $(11.25 \%)$ y tristeza con descanso $(9.75 \%)$.

\section{Tiempo de reacción}

Como medida de velocidad de procesamiento se utilizó el tiempo de reacción (TR). En promedio, el TR para todos los ensayos fue de 2.77 segundos $(D E=0.89$, Mediana $=$ 2.49). El ANOVA identificó un efecto principal significativo para Emoción $\left(F_{6,468}=42.08, p<.001, \eta_{p}^{2}=.35\right)$. Las expresiones de alegría tuvieron los menores TR en comparación con las expresiones de sorpresa, asco, ira, tristeza y miedo (todas las $p<.036$ ). Las expresiones de descanso y de sorpresa tuvieron menores TR que las expresiones de asco, ira, tristeza y miedo (todas las $p<.001$ ), y las expresiones de asco y tristeza menores TR que las expresiones de miedo (ambas $p<.001)$. La interacción Grupo $\times$ Modelo también fue significativa $\left(F_{1,78}=6.87, p=.01, \eta_{p}^{2}=.08\right)$, cuando el modelo fue un hombre las mujeres respondieron con menor $\operatorname{TR}(M=2.56, D E=.68)$ que los hombres $(M=3.01$, $D E=1.15, p=.03)$.

La correlación entre porcentaje de precisión y TR fue negativa y significativa para todas las expresiones emocionales (todas las $p<.035$ ), a excepción de la expresión de miedo (ver tabla 2).

\section{Discusión}

El objetivo del presente estudio fue construir y validar un repositorio de imágenes de expresiones faciales con contenido emocional que pueda ser empleado en estudios de reconocimiento emocional en población colombiana. Los resultados sugieren que el conjunto final de 70 imágenes representa de forma precisa las distintas emociones básicas, tanto de rostros femeninos como masculinos, con rasgos fenotípicos de población colombiana.

Los resultados muestran un nivel de acierto alto (80.92\%) en la precisión de la respuesta, lo cual respalda la idoneidad de la imagen para expresar la emoción correspondiente y es superior a los porcentajes reportados en estudios similares, por ejemplo, las expresiones del NimStim, donde se encontró un índice de acierto del 79\% (Tottenham et al., 2009), el conjunto JACFEE que presentó un $74 \%$ de acierto (Biehl et al., 1997), el estudio de imágenes de afecto facial con un $88 \%$ (Ekman \& Friesen, 1971) y la validación de un grupo de imágenes del conjunto Karolinska Emotional Direct Faces con un 72\% (Goeleven, De Raedt, Leyman \& Verschuere, 2008).

La segunda medida de validez (coeficiente kappa de Fleiss) arrojó un puntaje categorizado por Polit et al. (2007) como bueno/excelente (Media $=.72$, Mediana $=.79)$. Lo anterior indica que existe una consistencia alta entre las etiquetas asignadas a las emociones y la emoción expresada. Los resultados coinciden con datos reportados en estudios similares, por ejemplo, en el instrumento NimStim se obtuvo un coeficiente de acuerdo entre participantes de .79 (Tottenham et al., 2009).

La expresión emocional con mayor porcentaje de acierto fue la alegría, y la de menor porcentaje, el miedo.

Tabla 1 Matriz de confusión (Media y DE) para el porcentaje de sujetos que eligió cada categoría de expresión emocional

\begin{tabular}{lrrrrrrr}
\hline \multicolumn{7}{c}{ Emoción seleccionada } \\
\hline \multicolumn{1}{c}{ Imagen } & \multicolumn{1}{c}{ Descanso } & \multicolumn{1}{c}{ Alegría } & \multicolumn{1}{c}{ Sorpresa } & \multicolumn{1}{c}{ Ira } & \multicolumn{1}{c}{ Asco } & \multicolumn{1}{c}{ Tristeza } & \multicolumn{1}{c}{ Miedo } \\
\hline Descanso & $\mathbf{9 6 . 7 5 ( 3 . 4 4 )}$ & $0(0.0)$ & $.25(.79)$ & $1.5(2.26)$ & $.25(.52)$ & $.25(.52)$ & $1.00(2.34)$ \\
Alegría & $2.12(4.67)$ & $94.87(5.47)$ & $.87(1.56)$ & $.12(.39)$ & $1.87(1.69)$ & $0(0.0)$ & $.12(.39)$ \\
Sorpresa & $.12(.39)$ & $1.75(2.58)$ & $\mathbf{9 3 . 5 ( 3 . 2 7 )}$ & $.12(.39)$ & $1.25(1.31)$ & $.12(.39)$ & $3.12(2.30)$ \\
Ira & $8(7.86)$ & $0(0.0)$ & $1.25(1.17)$ & $\mathbf{8 1 . 8 7 ( 1 0 . 0 7 )}$ & $3.37(4.29)$ & $2.87(4.16)$ & $2.62(3.03)$ \\
Asco & $.75(1.58)$ & $.25(.79)$ & $2.12(2.43)$ & $14.62(17.61)$ & $\mathbf{7 8 . 5 ( 1 8 . 3 5 )}$ & $1.5(1.64)$ & $2.25(3.42)$ \\
Tristeza & $9.75(16.88)$ & $.25(.52)$ & $1.75(3.07)$ & $.87(.84)$ & $6.75(8.70)$ & $\mathbf{6 8 . 2 5 ( 1 7 . 0 1 )}$ & $12.37(8.50)$ \\
Miedo & $3(3.82)$ & $.12(.39)$ & $27(15.65)$ & $1.62(2.43)$ & $4.25(5.95)$ & $11.25(18.41)$ & $\mathbf{5 2 . 7 5 ( 1 2 . 2 3 )}$ \\
\hline
\end{tabular}

En negrillas la selección correcta. 
Tabla 2 Coeficiente de correlación de Pearson $\left(R^{2}\right)$ para porcentaje de acierto de la respuesta y tiempo de reacción para cada emoción

Tiempo de respuesta

\begin{tabular}{|c|c|c|c|c|c|c|c|}
\hline$\%$ de acierto & Descanso & Alegría & Sorpresa & Ira & Asco & Tristeza & Miedo \\
\hline Descanso & $-.41^{* * *}(.16)$ & & & & & & \\
\hline Alegría & & $-.37^{* *}(.13)$ & & & & & \\
\hline Sorpresa & & & $-.28^{*}(.07)$ & & & & \\
\hline Ira & & & & $-.40^{\text {*** }}(.16)$ & & & \\
\hline Asco & & & & & $-.32^{* *}(.10)$ & & \\
\hline Tristeza & & & & & & $-.23^{*}(.05)$ & \\
\hline Miedo & & & & & & & $.01(.00)$ \\
\hline
\end{tabular}

Nota: ${ }^{*}<.05,{ }^{* *}<.001, R^{2}$ : proporción de la varianza total explicada.

Resultados similares han sido encontrados en la mayoría de estudios previos en este campo. Por ejemplo, Tottenham et al. (2009), encontraron para alegría $92 \%$ y para miedo $47 \%$; Goeleven et al. (2008), 92\% para alegría y 43\% para miedo; Dodich et al. (2014), 98\% para alegría y 62\% para miedo. Sin embargo, en el reconocimiento de estímulos del conjunto JACFEE, se encontraron mayores porcentajes de acierto en miedo en países como Estados Unidos (79\%). Esta leve discrepancia en los resultados podría explicarse a partir de las diferencias en las reglas aprendidas culturalmente respecto a los juicios emocionales (Biehl et al., 1997).

El acierto en el reconocimiento de la alegría ha sido relacionado con la influencia de la exposición cotidiana a la expresión de esta emoción, mientras que el miedo es una emoción menos frecuente. Además, la complejidad de la emoción varía en función de los músculos implicados en su expresión, razón por la que la alegría es considerada una emoción simple y el miedo una emoción compleja (Biehl et al., 1997). Otras explicaciones han planteado que las microexpresiones del miedo podrían sobreponerse con las expresiones de otras emociones, en especial, sorpresa, lo que afectaría la precisión en su reconocimiento (Jack, Garrod \& Schyns, 2014), de hecho, en la presente investigación, se encontró que en el $27 \%$ de las evaluaciones se confundió la emoción de miedo con sorpresa. La variabilidad de acierto entre las distintas categorías emocionales se atribuye a las diferencias en el proceso de reconocimiento emocional y no a las características de la imagen (Tottenham et al., 2009).

La correlación entre precisión y velocidad de respuesta mostró que, para todas las expresiones, a excepción del miedo, una mayor precisión en el reconocimiento de la emoción se asoció con un menor tiempo de reacción. La relación entre latencia de la respuesta y precisión es un indicador de la complejidad de la tarea (i.e., mayor tiempo de respuesta se relaciona con mayor complejidad) y de una mayor demanda cognitiva (Wells, Gillespie \& Rotshtein, 2016). Los resultados del presente estudio sugieren que los estímulos del REFEC poseen un nivel de complejidad que favorece la velocidad de procesamiento en tareas de reconocimiento emocional.

Las comparaciones por sexo mostraron mayores porcentajes de acierto en el reconocimiento emocional en las participantes mujeres. Estas diferencias en las habilidades de reconocimiento emocional coinciden con estudios previos (Ma, Correll \& Wittenbrink, 2015). Estos resultados han sido explicados a partir de una mayor activación de estructuras límbico-subcorticales del lóbulo temporal en las mujeres, en comparación con los hombres, lo que se relacionaría con una mejor velocidad y precisión en el reconocimiento emocional asociado a ventajas evolutivas en el cuidado de la descendencia (Whittle, Simmons \& Allen, 2017).

En cuanto al factor modelo se encontró un mayor porcentaje de acierto en el reconocimiento de las emociones de sorpresa, asco y miedo expresadas por mujeres; no obstante, la expresión de ira en el modelo hombre generó un mayor porcentaje de acierto. Las mujeres han mostrado mayor intensidad en la expresión de emociones como el miedo (Wells et al., 2016), aspecto que se ha vinculado con conductas que representarían una ventaja evolutiva para la formación de lazos sociales (Hedger, Adams \& Garner, 2015). En el caso de los hombres, la clara expresión de emociones asociadas con dominancia y jerarquía interpersonal, como la ira, puede tener también ventajas evolutivas (Whittle et al., 2017).

La mayor capacidad de reconocimiento y expresión emocional en las mujeres también podría explicarse desde una postura ontogenética, que sugiere que las mujeres reciben desde la infancia una mayor estimulación frente a la conducta prosocial, lo cual favorece su entrenamiento en estas habilidades (Ellemers, 2018).

En conjunto, los resultados del presente estudio permiten concluir que el REFEC es un banco de imágenes de expresiones emocionales válido para estudios de reconocimiento emocional y, en general, para estudios que requieran estímulos de rostros emocionales en población colombiana. El uso de cada una de las expresiones emocionales debe tener en cuenta los porcentajes de acierto en la respuesta presentados en el material suplementario en línea, ya que cada expresión y modelo tienen diferentes puntajes.

Los resultados encontrados deben ser analizados considerando las siguientes limitaciones. En primer lugar, los modelos que participaron en el estudio fueron en su mayoría adultos jóvenes, aspecto que limita la representatividad de las diferentes etapas del ciclo vital. 
Otras investigaciones podrán utilizar la metodología aquí descrita para desarrollar un banco de imágenes de expresiones emocionales de niños y adultos mayores. Segundo, el rango de edad de los participantes fue de 18 a 35 años, por lo tanto, se sugiere que en próximos estudios se proporcionen datos en niños, adolescentes y adultos mayores. Futuras investigaciones podrían contemplar muestras más grandes y variadas. Tercero, Colombia es un país caracterizado por una amplia diversidad cultural y étnica. Si bien el presente estudio tuvo en cuenta los aspectos étnicos y culturales que representan al colombiano promedio, es necesario señalar que el banco de imágenes obtenido no representa la totalidad del universo étnico y cultural que conforma al pueblo colombiano. En este sentido, harían falta nuevos estudios que permitieran recoger dicha diversidad.

\section{Agradecimientos}

Agustín Patiño Orozco, quien desempeñó el rol de fotógrafo y editor, Ancízar Valencia por la construcción del código de programación del segundo estudio y al Grupo de Investigación en Psicología Cognitiva por el préstamo de insumos y espacios.

\section{Financiación}

Esta investigación fue financiada, parcialmente, por el Fondo para la Financiación de Trabajos de Grado, integrado por el Centro de Investigaciones de la Facultad de Ciencias Sociales y Humanas - Universidad de Antioquia- (CISH) y el Departamento de Psicología de la Universidad de Antioquia.

\section{Conflicto de intereses}

Los autores manifiestan no tener conflicto de intereses.

\section{Referencias}

Almanasreh, E., Moles, R., \& Chen, T. F. (2019). Evaluation of methods used for estimating content validity. Research in Social and Administrative Pharmacy, 15(2), 214-221. https://doi.org/ 10.1016/j.sapharm.2018.03.066

Barrett, L. F., Adolphs, R., Marsella, S., Martinez, A. M., \& Pollak, S. D. (2019). Emotional expressions reconsidered: Challenges to inferring emotion from human facial movements. Psychological Science in the Public Interest, 20(1), 1-68. https://doi. org/10.1177/1529100619832930

Biehl, M., Matsumoto, D., Ekman, P., Hearn, V., Heider, K., Kudoh, T., \& Ton, V. (1997). Matsumoto and Ekman's Japanese and Caucasian Facial Expressions of Emotion (JACFEE): Reliability data and cross-national differences. Journal of Nonverbal Behavior, 21(1), 3-21. https://doi.org/10.1023/A:1024902500935

Bland, J. M., \& Altman, D. G. (1995). Multiple significance tests: the Bonferroni method. BMJ, 310(6973), 170. https://doi. org/10.1136/bmj.310.6973.170

Chen, C., \& Jack, R. E. (2017). Discovering cultural differences (and similarities) in facial expressions of emotion. Current Opinion in Psychology, 17, 61-66. https://doi.org/10.1016/j. copsyc. 2017.06.010

Dodich, A., Cerami, C., Canessa, N., Crespi, C., Marcone, A., Arpone, M., ... \& Cappa, S. F. (2014). Emotion recognition from facial expressions: a normative study of the Ekman 60-Faces Test in the Italian population. Neurological Sciences, 35(7), 1015-1021. https://doi.org/10.1007/s10072-014-1631-x
Ekman, P. (1993a). Facial expression and emotion. American Psychologist, 48(4), 384-392. https://doi.org/10.1037/0003-066X.48.4.384

Ekman, P. (1993b). Pictures of facial affect (POFA). Oakland, CA: Author. Recuperado de www.paulekman.com

Ekman, P., \& Cordaro, D. (2011). What is meant by calling emotions basic. Emotion Review, 3(4), 364-370. https://doi. org/10.1177/1754073911410740

Ekman, P., \& Friesen, W. V. (1971). Constants across cultures in the face and emotion. Journal of Personality and Social Psychology, 17(2), 124-129. https://doi.org/10.1037/h0030377

Ellemers, N. (2018). Gender stereotypes. Annual Review of Psychology, 69, 275-298. https://doi.org/10.1146/annurev-psych-122216-011719

Fleiss, J. L. (1981). Statistical methods for rates and proportions. Nueva York: John Wiley and Sons.

Gantiva, C., Barrera-Valencia, M., Cadavid-Ruiz, N., Calderón-Delgado, L., Gelves-Ospina, M., Herrera, E., ... \& Suárez-Pico, P. (2019). Induction of Affective States with Pictures: Second Colombian Validation of the International Affective Pictures System (IAPS). Revista Latinoamericana de Psicología, 51(2), 176195. https://doi.org/10.14349/rlp.2019.v51.n2.5

Gantiva, C., Sotaquirá, M., Araujo, A., \& Cuervo, P. (2020). Cortical processing of human and emoji faces: an ERP analysis. Behaviour \& Information Technology, 39(8), 935-943. https://doi.org/ 10.1080/0144929X.2019.1632933

Goeleven, E., De Raedt, R., Leyman, L., \& Verschuere, B. (2008). The Karolinska directed emotional faces: a validation study. Cognition and Emotion, 22(6), 1094-1118. https://doi. org/10.1080/02699930701626582

Hedger, N., Adams, W \& Garner, M. (2015). Hedger, N., Adams, W. J., \& Garner, M. (2015). Fearful faces have a sensory advantage in the competition for awareness. Journal of Experimental Psychology: Human Perception and Performance, 41(6), 17481757. https://doi.org/10.1037/xhp0000127

Jack, R. E., Garrod, O. G., \& Schyns, P. G. (2014). Dynamic facial expressions of emotion transmit an evolving hierarchy of signals over time. Current Biology, 24(2), 187-192. https://doi.org/ 10.1016/j.cub.2013.11.064

Jeanneret, G., Oña, A., Rego, P., Vaiman, M., \& Pereno, G. (2015). Estudio bibliométrico de publicaciones científicas que utilizan pruebas de reconocimiento de emociones faciales. Anales de Psicología, 31(1), 324-337. https://doi.org/10.6018/analesps.31.1.158121

Lundqvist, D., Flykt, A., \& Öhman, A. (1998). The Karolinska directed emotional faces (KDEF). CD ROM from Department of Clinical Neuroscience, Psychology Section, Karolinska Institutet, 91, 630.

Ma, D.S., Correll, J., \& Wittenbrink, B. (2015). The Chicago face database: A free stimulus set of faces and norming data. Behavior Research Methods, 47(4), 1122-1135. https://doi.org/10.3758/ s13428-014-0532-5

Matsumoto, D., \& Ekman, P. (1988). Japanese and Caucasian facial expressions of emotion (JACFEE) [Slides]. San Francisco, CA: Intercultural and Emotion Research Laboratory, Department of Psychology, San Francisco State University.

Narambuena, L., Vaiman, M., \& Pereno, G. L. (2016). Reconocimiento de emociones faciales en adultos mayores de la ciudad de Córdoba. Psykhe, 25(1), 1-13. https://doi.org/10.7764/ psykhe.25.1.791

Polit, D. F., Beck, C. T., \& Owen, S. V. (2007). Is the CVI an acceptable indicator of content validity? Appraisal and recommendations. Research in Nursing \& Health, 30(4), 459-467. https://doi. org/10.1002/nur.20199

Peirce, J. W. (2009). Generating stimuli for neuroscience using PsychoPy. Frontiers in Neuroinformatics, 2, 10. https://doi. org/10.3389/neuro.11.010.2008 
Quintero, C., García, J., Muñoz, C., Rangel, A., Palacio, C., Ospina-Duque, J., \& Rodríguez-Losada, J. (2015). Sensibilidad en el reconocimiento de emociones faciales como endofenotipo de esquizofrenia. Revista Colombiana de Psicología, 24(1), 113-127. https://doi.org/10.15446/rcp.v24n1.41738

Sharma, U., Desikachari, B. R., \& Sarma, S. (2019). Content validity of the newly developed risk assessment tool for religious mass gathering events in an Indian setting (Mass Gathering Risk Assessment Tool-MGRAT). Journal of Family Medicine and Primary Care, 8(7), 2207-2211. https://doi.org/10.4103/jfmpc. jfmpc_380_19

Suárez-Pico, P., Bonelo-Cuellar, G., \& Utria, O. (2019). Diseño de un software para estimulación del componente socioemocional en niños con trastornos del espectro autista. Psychologia, 13(1), 111-124. https://doi.org/10.21500/19002386.4080

Torrado, D., Ornar, E., Prada, S., Edward, L., \& Santos, A., (2012). Análisis psicométrico del Cuestionario de Reconocimiento de Emociones Faciales (CREF): indicadores en población colombiana. Pensamiento Psicológico, 10(2), 103-112.

Tottenham N., Tanaka, J., Leon, A., McCarry, T., Nurse M., \& Nelson, C. (2009). The NimStim set of facial expressions: Judgments from untrained research participants. Psychiatry Research, 168, 242-249. https://doi.org/10.1016/j.psychres.2008.05.006
Wells, L. J., Gillespie, S. M., \& Rotshtein, P. (2016). Identification of emotional facial expressions: Effects of expression, intensity, and sex on eye gaze. PloS One, 11(12), e0168307. https://doi. org/10.1371/journal.pone.0168307

Whittle, S., Simmons, J. G., \& Allen, N. B. (2017). Emotion and Gender-Specific Neural Processing in Men and Women. En M. J. Legato (Ed.), Principles of Gender-Specific Medicine (pp. 183-201). Academic Press. https://doi.org/10.1016/b978-0-12-8035061.00013-9

Wilks, C. R., Morland, L. A., Dillon, K. H., Mackintosh, M. A., Blakey, S. M., Wagner, H. R., \& Elbogen, E. B. (2019). Anger, social support, and suicide risk in US military veterans. Journal of Psychiatric Research, 109, 139-144. https://doi.org/10.1016/j.jpsychires.2018.11.026

World Medical Association Declaration of Helsinki (2000). JAMA, the Journal of the American Medical Association, 284, 3043-3045. https://doi.org/10.1001/jama.284.23.3043

Young, A., Perrett, D., Calder, A., Sprengelmeyer, R., \& Ekman, P. (2002). Facial expressions of Emotion - Stimuli and Tests (FEEST). Bury St. Edmunds, England: Thames Valley Test. 